Orthopäde $2010 \cdot 39: 1097$

DOI 10.1007/s00132-010-1684-3

Online publiziert: 25. November 2010

(c) Springer-Verlag 2010

M. Engelhardt $\cdot 0$. Lorbach

Klinik für Unfall-, Hand- und orthopädische Chirurgie, Klinikum Osnabrück

\title{
Muskel- und Sehnenverletzungen
}

Sehnenverletzungen können akut auftreten, vollziehen sich aber überwiegend auf dem Boden degenerativer Veränderungen. Sie können mit einem deutlichen Funktionsverlust einhergehen, bei denen dann eine schnelle operative Rekonstruktion angestrebt wird. Falls die Verletzung zu keinem starken Funktionsverlust führt, wird insbesondere beim Freizeitsportler eher eine konservative Behandlung eingeleitet.

\section{(7) 10-50\% der Verletzungen im Sport sind Muskelverletzungen}

Muskelverletzungen beinhalten 10-50\% der Verletzungen im Sport. Dieses Thema ist von besonderem Interesse, da hier nur wenige hochwertige Studien existieren und die Behandlungsstrategien sich eher auf Erfahrungsberichte und empirische Daten stützen. Im Gegensatz zu Verletzungen im Sehnenbereich, welche falls notwendig, in der Regel gut refixiert werden können, sind bei intra- und intermuskulären Verletzungen häufig nur adaptierende Fasziennähte möglich.

Die vorliegende Ausgabe befasst sich mit sportspezifischen Verletzungen der Muskeln und Sehnen. Neben den Rupturen des Lig. patellae und des M. quadriceps femoris, sowie den proximalen und distalen Rupturen der Bizepssehne am Oberarm soll das Heft auch einen Einblick über die Behandlung der weniger häufig vorkommenden Verletzungen der ischiokruralen Muskulatur, des Tibialis anterior und der Peronäalsehnen geben. Die Beugesehnenverletzungen der Hand sind bei Klettersportlern immer wieder zu sehen und dürfen daher nicht fehlen.
Das Themenheft wird durch die Behandlung der muskulären Verletzungen abgerundet, mit denen insbesondere Ärzte, die in der Teambetreuung tätig sind, regelmäßig konfrontiert werden.

Wir hoffen, dass die ausgearbeiteten Beiträge dieses Heftes dem ambitionierten Orthopäden, Unfallchirurgen und Sportarzt in der täglichen Praxis von Nutzem sind.

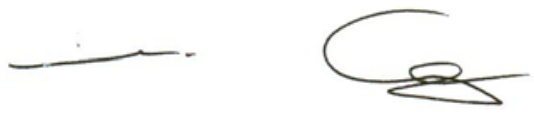

M. Engelhardt

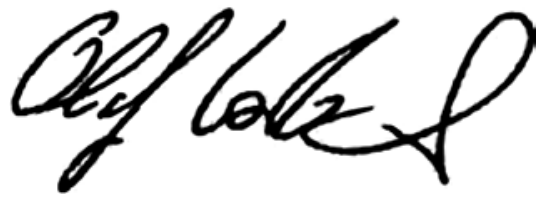

O. Lorbach

Korrespondenzadresse

PD Dr. M. Engelhardt

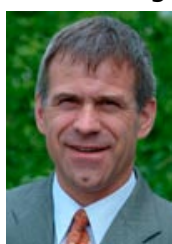

Klinik für Unfall-, Hand- und orthopädische Chirurgie, Klinikum Osnabrück Am Finkenhügel 1-3, 49076 Osnabrück martin.engelhardt@ klinikum-os.de

\section{Dr. O. Lorbach}

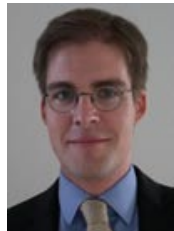

Klinik für Unfall-, Hand- und orthopädische Chirurgie, Klinikum Osnabrück Am Finkenhügel 1-3, 49076 Osnabrück olaf.lorbach@klinikum-os.de 